\title{
Grand challenge in livestock genomics: for food, for medicine, for the environment, for knowledge
}

\author{
Guilherme J. M. Rosa ${ }^{1,2 *}$ \\ Department of Animal Sciences, University of Wisconsin - Madison, Madison, WI, USA \\ 2 Department of Biostatistics and Medical Informatics, University of Wisconsin-Madison, Madison, WI, USA \\ *Correspondence: grosa@wisc.edu
}

Frontiers in Livestock Genomics (FLG) provides a venue for investigators to publish basic, applied, and methodological research toward a better understanding of the genetic mechanisms underlying the expression of complex phenotypes in livestock, the effects of domestication and evolutionary processes on genome variability, as well as innovative strategies for the genetic improvement of economically and socially important traits in livestock. The terms "livestock" and "genomics" are used here with broad, inclusive definitions. By livestock we refer to animal species raised in agricultural settings to produce commodities such as food, fiber, and labor. In this context, livestock species span from mammals (e.g., cattle, sheep, goat, horses, and pigs) to birds (e.g., poultry), fish, and crustaceans (e.g., farmed fish and shrimp), and even to some species of insects (e.g., honeybees and silkworm), mollusks (e.g., escargot snail), and amphibians (e.g., frogs). By "genomics" we refer to a broad field of genetics and molecular biology concerning the study of genomes, such as DNA sequence and fine-scale genetic mapping efforts, and also functional genomics efforts to try to elucidate gene functions and interactions, and how they relate to phenotypic expression. In addition, the term "genomics" in the title of this Specialty Section of Frontiers in Genetics encompasses investigation aiming efficient use of the knowledge generated by genetics and genomics studies for the improvement of yield and quality of animal products, health and well-being of livestock, as well as the sustainability of animal production systems.

\section{THE IMPORTANCE OF LIVESTOCK SPECIES FOR HUMAN POPULATIONS}

Some livestock species have been domesticated thousands of years ago, and have since been evolving together with human populations. Through selective breeding over the years livestock species have been successfully improved in terms of yield and production efficiency (Muir and Aggrey, 2003; Rosa, 2011), allowing better use of land resources, including areas that are not suitable for other agricultural purposes. Nowadays, farm animals supply human populations with food (e.g., meat, milk, and honey), raw material for clothing, furniture, and other goods (e.g., leather, silk, and feather), labor (e.g., transportation, power, pollination), and companionship. Unquestionably, farm animals represent a vital social and economic component for human communities (Rothschild and Plastow, 2007). However, the world has been undergoing continued population growth, as well as rising incomes and urbanization, especially in developing countries. Such social changes are reflected on a rapidly growing demand for meat and other animal products. The challenge is even worsened as supplying with such a growing demand should comply with moral and economic constrains related to animal well-being issues and sustainability of agricultural enterprises, as well as climate change.

In addition to providing food, fiber, and labor, livestock species also contribute significantly with human medicine (Lewin, 2009; Roberts et al., 2009). For example, chicken eggs are used intensively in the development and production of vaccines, and horses are utilized in the production of snake antivenom. In addition, xenotransplantation (i.e., the transplantation of cells, tissue, or organs between species) may represent a potential way in which animals might also contribute with human health. As there is a worldwide shortage of organs for clinical implantation, some research has been developed for studying the possibility of using animals' tissues and organs for transplantation into human patients with end-stage organ failure. A preferred species in this regard is the pig, given its comparable size and physiology, and availability (Rothschild and Plastow, 2007).
Nonetheless, besides legal and ethical issues, further investigation is still necessary for routine and successful xenotransplantation, to overcome tissue rejection response and potential disease transmission, among other issues.

\section{LIVESTOCK SPECIES AS MODELS FOR HUMAN RESEARCH}

Selective breeding during the domestication process, together with population isolation and bottlenecks, has led to different lines and breeds within livestock species. Such breeds present variable population structures and inbreeding levels, and were subjected to different selection intensities targeting different traits, culminating with a remarkable intra-specific spectrum of phenotypic variability and genome diversity. Such genetic and phenotypic diversity in livestock species make them an extremely valuable resource as model species for human genetic studies (de Koning et al., 2007). Indeed, a number of biomedical research endeavors have used livestock species in different contexts and application goals, from comparative physiology and genomics, to cloning, artificial insemination, and production of pharmaceuticals. Advantages of livestock over other animal species as model organisms for biomedical research include their size (especially important for example in research on surgery procedures), relatively long generation interval, comparable physiology, and availability. Alternative species and breeds within species can be used for different applications, for example uniparous species such as the cow are interesting for human reproductive research, and pigs have been used on obesity research. In addition, some livestock models have been developed for specific applications, such as the CFTR gene knockout pig, which is a useful model for studies regarding cystic fibrosis, and the baby pig, which is the model of choice for research on human infant nutrition. 
More specifically in the context of genetics and genomics, livestock species present the advantage of being outbred populations, like humans, and with larger families, full pedigree information, and allowing various mating designs. In addition, reproductive technologies can be routinely employed, and environmental factors can be easily recorded or controlled. Some livestock species have been already fully sequenced, and their genomes have been undergoing continuous curation and annotation (Womack, 2005). In addition, highdensity single nucleotide polymorphism (SNP) panels are already available for some species, especially cattle, chicken, and pig, allowing whole genome association analysis for routinely recorded traits, as well as for phenotypes measured in experimental settings. Hence, through comparative genomics techniques, livestock studies can provide insight into evolutionary processes in natural populations, and contribute to a better understanding of the genetic architecture underlying disease and other complex traits in humans (Womack, 2005; de Koning et al., 2007).

\section{CURRENT AND FUTURE CHALLENGES}

Notable genetic improvement of desirable traits has been observed in livestock species. Such genetic changes have been accomplished mostly through the selection and breeding of superior animals using principles of quantitative and population genetics (Rosa, 2011). Three scientific-technological innovations were fundamental for the acceleration of genetic improvement rates, especially in beef and dairy cattle, chickens, and pigs: (1) the advent of reproductive technologies, such as the artificial insemination; (2) the development of efficient statistical models to analyze complex and unbalanced field data, by simultaneously accounting for genetics and environmental effects affecting phenotypic traits, as well as pedigree information and correlations among traits; and (3) the arrival of modern computers, with enough memory and processing power to handle and implement such data sets and statistical models. In addition, in the last two decades or so, with the advent of genotyping tools for molecular markers such as microsatellites, much interest sparked for gene mapping, and for incorporating such information into breeding programs using marker assisted selection techniques
(Dekkers and Hospital, 2002; Andersson and Georges, 2004; de Koning et al., 2007; Goddard and Hayes, 2007).

Together with genotyping tools, lowthroughput techniques became available also for sequencing small DNA fragments and for profiling gene expression, even in a small scale, allowing the first functional genomics studies. The technology has evolved rapidly and nowadays highthroughput techniques exist for example for simultaneous expression profiling of the entire genome, using microarrays or RNAseq tools, and for genotyping highdensity panels of molecular markers such as SNPs. Such technology has been used intensively in human research and studies with model organisms, and similar trend has been observed with livestock research as well (Womack, 2005).

However, with the increasing demand for food and other animal products worldwide, concomitantly with agriculture sustainability, animal well-being, and environmental concerns, livestock production is faced with an enormous challenge. Sustainable increase of yield and quality of animal products requires a coordinated action of the various aspects of animal production enterprises and their relationship with human communities, including animal nutrition, management, and reproduction, as well as ecological, environmental, and societal components. In this context, genomics is an especially important element, providing tools and resources for studies to elucidate genetic mechanisms controlling phenotypic traits, and for the development of novel selection strategies that can accelerate the genetic improvement rate in livestock (Rothschild and Plastow, 2007). In addition to the direct impact in agriculture, livestock genomics contributes also toward our understanding of evolutionary processes in natural populations, the relationships between genome variability and phenotypic traits in other species through comparative genomics, and as a potential source of human medicines (de Koning et al., 2007; Lewin, 2009; Roberts et al., 2009).

Genomics tools and technology are at different stages of development for different livestock species. For the more commonly studied species such as cattle, chicken, pig, and horse there are already many tools and resources available, such as genome sequence, annotation databases, genotyping, and expression profiling chips, etc. Yet for some other species genomic resources are in their very early stages of development. All livestock species benefit from genomic technology developments in humans and model organisms (Womack, 2005), but ultimately species-directed funding and coordinated research by the scientific community is required for the development of specific tools and resources to be used in livestock, such as genome sequencing for different species, detailed curation and annotation of genomes, development of genotyping and expression assay platforms, and the development of phenotype databases (Roberts et al., 2009). In addition, computational and statistical tools are necessary for an efficient storage, handling, and sharing of databases, as well as for an appropriate data-mining in terms of understanding the relationships among DNA polymorphisms and phenotypic variation, systems biology approaches to study gene and metabolic pathways and, finally, the development of accurate predictive models to estimate the genetic merit of animals (Goddard and Hayes, 2007), either for selection of superior animals or for individualized management strategies.

In this context, FLG offers a valuable forum for dissemination and discussion of scientific research findings on the development of genomics tools and their application in genomics studies and genetic improvement of livestock species to couple with the global challenge in terms of sustainable and ecologically sound production of high quality food and other animal products. Over two centuries ago, Malthus (1798) wrote "The capacity of improvement in plants and animals, to a certain degree, no person can possibly doubt. A clear and decided progress has already been made; and yet I think it appears, that it would be highly absurd to say, that this progress has no limits." Though Malthus may be correct in total, to date, the scientific use of breeding and genetics has continued to bring vast improvements and no limits have yet been reached. We look forward to studying and promoting this continued progress for generations to come!

\section{REFERENCES}

Andersson, L., and Georges, M. (2004). Domestic animal genomics: deciphering the genetics of complex traits. Nat. Rev. Genet. 5, 202-212.

de Koning, D.-J., Archibald, A., and Haley, C. S. (2007). Livestock genomics: bridging the gap between mice and men. Trends Biotechnol. 25, 483-489. 
Dekkers, J. C. M., and Hospital, F. (2002). The use of molecular genetics in the improvement of agricultural populations. Nat. Rev. Genet. 3, 22-32.

Goddard, M. E., and Hayes, B. J. (2007). Genomic selection. J. Anim. Breed. Genet. 124, 323-330.

Lewin, H. A. (2009). It's a bull's market. Science 324, 478-479.

Malthus, T. R. (1798). An Essay on the Principle of Population, 1st Edn. London: Penguin Classics. Available at: http://en.wikipedia.org/wiki/ An_Essay_on_the_Principle_of_Population

Muir,W.M., and Aggrey,S.E. (eds). (2003). Poultry Genetics, Breeding and Biotechnology. Oxfordshire: CABI.
Roberts, R. M., Smith, G. W., Bazer, F.W., Cibelli, J., Seidel, G. E. Jr., Bauman, D. E., Reynolds, L. P., and Ireland, J. J. (2009). Farm animal research in crisis. Science $324,468-469$.

Rosa, G. J.M. (2011)."Foundations of animal breeding," in Encyclopedia of Sustainability Science and Technology, ed. R. A. Meyers (Springer). (in press). Available at: http:// www.springer.com/physics/book/978-1-4419-0851-3 Rothschild, M. F., and Plastow, G. S. (2007). Impact of genomics on animal agriculture and opportunities for animal health. Trends Biotechnol. 26, 21-25.

Womack, J. E. (2005). Advances in livestock genomics: opening the barn door. Genome Res. 15, 1699-1705.
Received: 16 May 2011; accepted: 03 June 2011; published online: 11 July 2011.

Citation: Rosa GJM (2011) Grand challenge in livestock genomics: for food, for medicine, for the environment, for knowledge. Front. Gene. 2:34. doi: 10.3389/ fgene.2011.00034

This article was submitted to Frontiers in Livestock Genomics, a specialty of Frontiers in Genetics.

Copyright $\odot 2011$ Rosa. This is an open-access article subject to a non-exclusive license between the authors and Frontiers Media SA, which permits use, distribution and reproduction in other forums, provided the original authors and source are credited and other Frontiers conditions are complied with. 